\title{
Prevalent Causes of Mortality in the Iranian Population
}

\author{
Amir Hossain Mirhashemi ${ }^{1}$, Mohammad Hossain Kalantar Motamedi ${ }^{2}$, Sedigheh Mirhashemi ${ }^{*}$, \\ Hamidreza Taghipour ${ }^{2}$, Zahra Danial ${ }^{2}$
}

\author{
${ }^{1}$ Tehran University of Medical Sciences, Tehran, Iran \\ ${ }^{2}$ Trauma Research Center, Baqiyatallah University of Medical Sciences, Tehran, Iran
}

*Corresponding Author: Sedigheh Mirhashemi, M.D., Trauma Research Center, Baqiyatallah University of Medical Sciences, Tehran, Iran. Tel: +98-2188053766, Fax: +98-2188053766, Email: sedmir54@yahoo.com

Received February 19, 2017; Accepted May 6, 2017, Online Published July 5, 2017

\section{Dear Editor,}

The prevalence of death and changes to it in different geographical regions makes identifying cause of death (COD) an important issue in many instances. Based on data from the World Health Organization (WHO), noncommunicable diseases (NCDs) will account for $70 \%$ of all deaths by $2030 .{ }^{1}$ It seems that cardiovascular diseases, cancers, and motor vehicle accidents are the major causes of death in the Iranian population, with maximum years of life lost due to motor vehicle accidents, cardiovascular diseases and cancers, respectively.

In one study, cardiovascular diseases in 95 cases (26.4\%), motor vehicle accidents in $64(17.8 \%)$, cancers in $41(11.4 \%)$, inadvertent trauma in $23(6.4 \%)$, intentional trauma in 22 (6.1\%), stroke in $10(2.8 \%)$, lower respiratory infections in 8 (2.2\%), diabetes in $6(1.8 \%)$, and other causes in $91(25.3 \%)$ cases accounted for COD. Cardiovascular diseases rank first. ${ }^{1}$ In East Asia, the burden of cardiovascular diseases ranks second. ${ }^{2}$ Similarly, death rates due to motorcycle accidents are greater than twice compared to the global average. Some studies reported that road traffic accidents have about 28000 mortality rates annually in Iran, and damage or incapacitate 300000 . So, every one hour, about three people die on Iran's roads. Also, every two minutes people will be informed that one of their near friends has survived an accident but with severe damage and possibly long lasting injury. Another study reported that injuryrelated mortality will reach to 8.4 million in 2020 , rising from 5.1 in 1990 with traffic accidents being the leading reason. ${ }^{3}$ In recent years, the incidence of injuries and its dependent mortality have increased dramatically in Iran. The preventive measures subsequently suggested were the improvement of road safety and quality and safety of vehicles and the implementation of stringent laws for violators. Moreover, better coordination of police, fire department, and emergency departments is warranted to improve and expedite emergency service delivery to victims. This management may reduce arrival time to the scene and render critical intermediation more rapidly. Cancers rank third as a cause of mortality, ${ }^{1}$ and smoking plays a major role. Air pollution from fossil fuels, exposure to occupational pollutants, drug usage, and diet are other risk factors for cancers. ${ }^{4}$ Sudden natural death (SUND) is one of the causes of youth mortality. Stressful lifestyle, bad food habits, air pollution, smoking, and heart diseases are predicted to play an important role in SUND of Iranian individuals.

The government intends to promote methodical knowledge, develop educational and cultural programs, and decrease high-risk behaviors in society to promote a healthy lifestyle. 5,6 The coordination of civil services and emergency medical services is warranted to improve and expedite emergency service delivery to victims.

\section{Author's Contributions}

All authors contributed equally to this paper.

\section{Conflict of Interest Disclosures}

No conflict of interest.

Ethical Approval

Not applicable.

\section{References}

1. Saadat S, Yousefifard M, Asady H, Jafari AM, Fayaz M, Hosseini $\mathrm{M}$. The most important causes of death in Iranian population; a retrospective cohort study. Emerg (Tehran). 2015;3(1):16-21.

2. World Health Organization. The 10 leading causes of death in the world, 2000 and 2012. http://www.who.int/mediacentre/ factsheets/fs310/en/. Published May 2014. Updated January 2017.

3. Montazeri A. Road-traffic-related mortality in Iran: a descriptive study. Public Health. 2004;118(2):110-113. doi:10.1016/ S0033-3506(03)00173-2

4. Forouzanfar MH, Sepanlou SG, Shahraz S, et al. Evaluating causes of death and morbidity in Iran, global burden of diseases, injuries, and risk factors study 2010. Arch Iran Med. 2014;17(5):304-320.

5. Fayaz AF, Goudarzi A, Taghaddosinejad F, Behnoush B. Sudden unexpected natural death in the youth; an Iranian single center Investigation. Int J Med Toxicol Forensic Med. 2014;5(1):8-11.

6. Winkel BG, Risgaard B, Bjune $T$, et al. Gender differences in sudden cardiac death in the young-a nationwide study. BMC Cardiovasc Disord. 2017;17:19. doi:10.1186/s12872-0160446-5.

Copyright $\odot 2017$ The Author(s). This is an open-access article distributed under the terms of the Creative Commons Attribution License (http:// creativecommons.org/licenses/by/4.0), which permits unrestricted use, distribution, and reproduction in any medium, provided the original work is properly cited. 